\title{
Young supernova remnants and the knee in the cosmic ray spectrum
}

\author{
A. Erlykin ${ }^{1}$, T. Wibig ${ }^{2}$, and A. W. Wolfendale ${ }^{3}$ \\ ${ }^{1}$ Lebedev Physical Institute, Moscow 117924, Russia \\ ${ }^{2}$ Physics Dept., University of Łódź; Sołtan Inst. Nucl. Studies, Łódź, Poland \\ ${ }^{3}$ Department of Physics, Durham University, Durham, UK
}

Received: 6 November 2010 - Revised: 20 January 2011 - Accepted: 24 January 2011 - Published: 19 May 2011

\begin{abstract}
It has recently been suggested that neutron stars inside the shells of young supernova remnants (SNR) are the sources of $\mathrm{PeV}$ cosmic rays and that the interaction of the particles with the radiation field in the SNR causes electron pair production, which has relevance to recent observations of "high" positron fluxes. Furthermore, the character of the interaction is such that the well-known knee in the cosmic ray energy spectrum can be explained. Our examination of the mechanism leads us to believe that the required parameters of $\mathrm{SN}$ and pulsars are so uncommon that the knee and positron fraction can only be explained if a single, local and recent $\mathrm{SN}$ - and associated pulsar - are concerned. In this case the mechanism can be valid.
\end{abstract}

\section{Introduction}

Although it is over 50 years since the "Knee" in the cosmic ray (CR) spectrum, at about $3 \mathrm{PeV}$, was discovered, its origin is still the subject of controversy. Many have considered that it is simply due to Galactic Modulation, the entrapment of CR by Galactic Magnetic fields becoming increasingly inefficient above this energy (we refer to this model as "GM": Galactic modulation). We ourselves, however, favour a "single source model" in which a single, recent local supernova (SN) is responsible, e.g. Erlykin and Wolfendale (1997, 2001).

In Erlykin and Wolfendale (2004) it was proposed that there is a specific SNR associated with the pulsar PSR B0656+14 responsible for "the single source". This pulsar is of age $\sim 10^{5}$ years and has a present period of $385 \mathrm{~ms}$, (Thorsett et al., 2003).

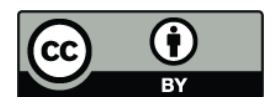

Correspondence to: $\mathrm{T}$. Wibig (wibig@zpk.u.lodz.pl)

\section{The pulsar, supernova remnant model}

We have argued that the knee is too sharp to allow the GM to work. This view has relevance to the recent work by $\mathrm{Hu}$ et al. (2009). which includes the observation of a sharp knee in the all-particle spectrum measured by the impressive Tibet AS- $\gamma$ array (Amenomori et al., 2008). It should be added that in Erlykin and Wolfendale (2001) an analysis was made of the results from 8 extensive air shower (EAS) arrays and comprising 19 shower size spectra. Later work (Erlykin and Wolfendale, 2011), using the results from 10 EAS arrays some being the same as used earlier but extending to higher energies - confirmed the earlier work on the sharp knee and claimed the existence of another knee (peak) at $80 \mathrm{PeV}$. This has relevance to both the SNR shock model and the mechanism to be described in that it gives support to the single source contention.

The advance made by Hu et al. (2009) is their proposal that pulsars $(\mathrm{P})$ close to their parent young supernovae $(\mathrm{SN})$ can give a spectrum of the appropriate shape, viz a sharp knee. We term the model: PSNR. The mechanism involved is cosmic ray nucleus-SN optical radiation interactions. A bonus is an explanation of recent excesses of electron/positron fluxes.

$\mathrm{Hu}$ et al. choose appropriate parameters and conclude that there are 3 possibilities; (1) all the sources are "standard", (2) the average effect is equivalent to using one set of parameters or (3) one single nearby source dominates the observed fluxes of CR in the knee region. In what follows we endeavor to determine which, if any, of these possibilities is valid. Insofar as they, and we, incline to the view that helium nuclei predominate at the knee, and, indeed, the knee is in the helium component itself, we concentrate on this component. In $\mathrm{Hu}$ et al. (2009) the parameters used are: an effective black body radiation temperature of $7000 \mathrm{~K}$, a period of acceleration of $\tau=0.19$ year with $n c \tau=12.9 \times 10^{29} \mathrm{~cm}^{-2}$ (where $n$ is the number of photons per $\mathrm{cm}^{3}$ in the interaction region). In the next section we examine the parameters in turn.

Published by Copernicus Publications on behalf of the Arbeitsgemeinschaft Extraterrestrische Forschung e.V. 


\section{Our analysis of the PSNR model}

\subsection{General remarks}

The parameters studied are:

The "sharpness" of the predicted helium spectrum.

The characteristics of pulsars.

The characteristics of young SN.

Concerning the CR spectral shape we have defined "sharpness", $S$, as the (negative) second differential of the logarithm of the intensity with respect to the logarithm of the energy. We have pointed out that the GM prediction is $S=0.3$; anything above this is regarded as needing a contribution from a sharply peaked (in log intensity times $E^{3}$ versus log energy) spectrum, derived from a single source. The EAS data give $\mathrm{S} \approx 2-3$ for the all particle spectrum, using $\Delta \log _{10} E=0.2$ in the derivation of $S$.

It is interesting to note that the analysis of data from the KASKADE EAS array (Apel et al., 2009) gave individual spectra for both protons and helium nuclei and for the latter we estimate $S \sim 2.5$, again a high value.

For the PSNR model of $\mathrm{Hu}$ et al. (2009) the predicted value is $S \sim 3.1$, a "high value" not inconsistent with the EAS data and the expectation for the single source model, particularly when the dilution effect of experimental errors is taken into account.

It remains to examine the reasonableness of the PSNR Model and, in particular to see whether "standard" sources of the type specified are likely to occur.

\subsection{Pulsars}

We start with the pulsars. Millisecond pulsars are involved, following the work of Gaisser et al. (1989). These workers give the relationship between the maximum (proton) energy available from pulsar acceleration as $E_{\max }=100 B_{12}$ $P_{10}^{2} \mathrm{PeV}$ where $B_{12}$ is the magnetic field in $10^{12}$ Gauss and $P_{10}$ is the period in units of $10 \mathrm{~ms}$. Thus, even a pulsar with period $100 \mathrm{~ms}$ should be able to reach several $\mathrm{PeV}$ if the magnetic field were somewhat bigger than $10^{12}$ Gauss. It is true that millisecond pulsars themselves account for about $3 \%$ of all pulsars but most of these are low magnetic field "spun-up" objects and the number of potentially useful millisecond pulsars is less than $1 \%$, (Lorimer et al., 2006). However those having $100 \mathrm{~ms}$ or less periods at birth are more frequent, although, very few have fields above the necessary $10^{12}$ Gauss (Lyne and Graham-Smith , 1990). It is just possible that our preferred "single source" pulsar (PSR B0656+14) was fast enough, and had a sufficiently high magnetic field at birth but the fraction of such pulsars must be very small and it is unlikely that they will give the whole of the PeV CR spectrum. The recently discovered "magnetastars" (Kasen and Bildsten, 2010), may provide the answer, however. These object seem to have periods in the range $2-20 \mathrm{~ms}$, magnetic fields $\sim 5 \times 10^{14}$ Gauss, and luminosities above about $10^{48} \mathrm{erg} \mathrm{s}^{-1}$. The bulk of their energy loss occurs over days to weeks. Their frequency may be as high as a few $\%$ so, again, the single source could be of this character but not the whole assembly of pulsars.

\subsection{Young SNR}

Turning to the associated young SN there are two potential problems to be considered: the "low" temperature of the SNR needed $(\sim 7000 \mathrm{~K})$ and the fact that the the temperature invariably falls during the expansion phase. Concerning the temperature during the phase when the young pulsar was injecting nuclei into it, there is uncertainty over its magnitude. Initially it was some $10^{6} \mathrm{~K}$ but quickly cooled reaching "optical temperatures" $\left(\sim 10^{4} \mathrm{~K}\right)$ when the environment became transparent. The " $7000 \mathrm{~K}$ " must be regarded as perhaps unlikely but possible. It should be added that some SNR emit copious fluxes of soft X-rays, eg $3 \times 10^{46} \mathrm{erg}$ over $1000 \mathrm{~s}$ for red supergiants (Nakar and Sari, 2010) which would interact with lower energy nuclei and cause complications; however this cannot be held against the model.

\subsection{Photon energy versus time}

Moving to the time dependence of the mean photon energy this needs examination. 3-D radioactive transfer calculations have been made by Kasen et al. (2010) and can be taken as an example. These workers quote mean temperatures as a function of time from the explosion as follows: $6 \times 10^{4} \mathrm{~K}$ (5 days), $3 \times 10^{4} \mathrm{~K}$ (10 days) and $2 \times 10^{4}$ (19 days). Multiplying by the radiation intensity as a function of time yields intensity times temperature for the times listed as 6,9 and 4 units. Thus, there is near constancy of effective intensity over a range of a factor 3 in temperature, with consequent smoothing of the knee in the derived CR spectrum.

Although the temperature here is higher than the $7000 \mathrm{~K}$ taken in $\mathrm{Hu}$ et al. (2009) the effect of temperature variation should be similar. Thus, we have examined in more detail the behavior of the sharpness of different CR mass components affected by the $e^{+} e^{-}$pair production mechanism for the model of an expanding SNR when the temperature varies by a factor of a few within several days. For the background photon energies of several $\mathrm{eV}$, and an initial energy density of $10^{15} \mathrm{eV} / \mathrm{cm}^{3}$ we found an effect above $10^{15} \mathrm{eV}$ specifically, a reduction in $S$ by a factor 1.6. This factor is not large and can, in fact, be probably disregarded.

\subsection{Sensitivity of sharpness to other parameters}

The sensitivity of the sharpness, $S$, to the spread of the other parameters involved needs examination. Other problems of the model, which are beyond present computation, include the following: 
i The effect of the shock wave of the SN, propagating at $\sim 3 \times 10^{4} \mathrm{~km} / \mathrm{s}$, on the emerging optical radiation and on the rotation characteristics of the pulsar, by way of the ensuing plasma environment (acceleration models usually neglect "viscosity").

ii Non-isotropic interactions between SN photons, which are strongly collimated, and the Pulsar-accelerated nuclei.

iii The big variation of luminosity, temperature and temperature vs time from one SNR to another.

iv The role of those particles which are still being generated by the pulsar after the SNR light has ceased.

In Erlykin and Wolfendale (2001), an analysis was made of $S$ as a function of the standard deviation $\sigma$ of the error in the logarithm of the energy and this can be used as an illustration of the effect of smoothing on the $S$-value. The form of the helium energy spectrum given in Hu et al. (2009) has been taken, and uncertainties in energy of magnitude $\sigma$ (in $\log E$ ) have been applied. Similar results to those in Erlykin and Wolfendale (2001) were derived; these have the following $S(\sigma)$ values with respect to unity at $\sigma=0$ : $0.6(0.1), 0.33(0.2)$ and, extrapolating, 0.1 at $\sigma=0.42$.

Returning to the $S$-value derived for Helium in $\mathrm{Hu}$ et al. (2009) (3.0), this resulted in a fit to the data for the all-particle spectrum with adequate sharpness, as already mentioned. We consider that a Helium $S$-value less than 1.0 would be quite unacceptable. From the above this indicates $\sigma \sim 0.2$, or, in linear scale, $\pm 60 \%$.

Applying these arguments to the model advanced in $\mathrm{Hu}$ et al. (2009) we have the following. To be acceptable there can be no deviation from the adopted parameters overall by more than about $\pm 60 \%$. This is not impossible for a particular single source and the PSNR model must be taken seriously.

\section{Relevance to electrons and positrons}

Mention must now be made of another important aspect of the PSNR model described in $\mathrm{Hu}$ et al. (2009): the excesses of electron and positron fluxes observed in a number of recent experiments. A "single nearby recent source" is able to give an explanation whether the mechanism is that in $\mathrm{Hu}$ et al. (2009) or the "single" SNR model, as described recently by Erlykin and Wolfendale (2011) following on their earlier work (Erlykin and Wolfendale, 2001). It should be added that in the single source model in which SNR shocks are responsible for accelerating the particles, these do not gain energy until some considerable time (many years) after the SN explosion so that losses due to interaction with the photon field are not important.

\section{Discussion and conclusions}

Further support for both single source models comes from the detection of the "iron peak" at some $80 \mathrm{PeV}$ (Erlykin and Wolfendale, 2011). In both cases iron nuclei are available for acceleration and their peak should be present. Another common aspect relates to the cosmic ray anisotropy. In Erlykin and Wolfendale (2006) we examine the whole question of the anisotropy of Galactic cosmic rays produced by stochastic SN explosions. The conclusion was that there is no inconsistency with the observed anisotropy; interestingly, if the identification of the single source with the SNR associated with PSR B0656+14 is correct, its excess cosmic ray flux may counteract the expected opposite particle flow and help to explain the measured low value for the anisotropy.

Finally, as applied to the origin of the knee in terms of many standard sources of the type specified in $\mathrm{Hu}$ et al. (2009), there appears to be no possibility at all. SN vary too much from one to the next and pulsars of the required characteristics appear to be too infrequent. Nevertheless, it seems possible that a single SNR, pulsar combination might have the required properties to allow the knee to be explained by the PSNR Model.

Edited by: R. Vainio

Reviewed by: two anonymous referees

\section{References}

Apel, W. D., Arteaga, J. C., Badea, A. F. et al.: Energy Spectra of Elemental Groups of Cosmic Rays: Update on the KASCADE Unfolding Analysis, Astropart. Phys., 31, 86 - 91, 2009.

Amenomori, M., Bi, X. J., Ciu, S. W. et al.” The All-Particle Spectrum of Primary Cosmic Rays in the Wide Energy Range from $10^{14}$ to $10^{17} \mathrm{eV}$ Observed with the Tibet-III Air-Shower Array, Astrophys. J., 678, 1165 - 1179, 2008.

Erlykin, A. D. and Wolfendale, A. W.: High energy cosmic ray spectroscopy. I. status and prospects, Astropart. Phys., 7, 1 - 13, 1997.

Erlykin, A. D. and Wolfendale, A. W.: Structure in the cosmic ray spectrum: An update, J. Phys. G: Nucl. Part. Phys., 27, 1005 1030, 2001.

Erlykin, A. D. and Wolfendale, A. W.: Supernova remnants and the origin of the cosmic radiation: the electroncomponent, J. Phys. G: Nucl. Part. Phys., 28, 359 - 378, 2002.

Erlykin, A. D., and Wolfendale, A. W.: Cosmic rays and the Monogem supernova remnant, Astropart. Phys., 22, 47 - 63, 2004.

Erlykin, A. D. and Wolfendale, A. W.: The anisotropy of galactic cosmic rays as a product of stochastic supernova explosions, Astropart. Phys., 25, 183 - 194, 2006.

Erlykin, A. D. and Wolfendale, A. W.: Do We See an Iron Peak?, ASTRA, 8, 1-5, 2011.

Gaisser, T. K., Harding, A. K., and Stanev, T.: Signatures of particle acceleration at SN 1987A, Astrophys. J., 345, 423 - 433, 1989. 
Hu, H-B., Yuan, Q., Wang, B., Fan, C., Zhang J.-L., Bi, X.-J.: On the e+e Excesses and the Knee of the Cosmic Ray SpectraHints of Cosmic Ray Acceleration in Young Supernova Remnants, Astrophys. J., 700, 170 - 173, 2009.

Kasen, D. and Bildsten, L.: Supernova Light Curves Powered by Young Magnetars, Astrophys. J., 717, 245 - 249, 2010.

Kasen, D., Thomas, R. C., and Nugent, P.: Time-dependent Monte Carlo Radiative Transfer Calculations for Three-dimensional Supernova Spectra, Light Curves, and Polarization, Astrophys. J., 651, 366 - 380, 2006.

Lorimer, D. R., Faulkener, A. J., Lyne, A. G. et al.: The Parkes Multibeam Pulsar Survey VI. Discovery and timing of 142 pulsars and a Galactic population analysis, Mon. Not. R. Astron. Soc., 372, 777 - 800, 2006.
Lyne, A. G. and Graham-Smith, F.: Pulsar Astonomy, CUP, Cambridge, UK 1990.

Nakar, E. and Sari, R.: Early supernovae light-curves following the shock breakout, Astrophys. J., 725, 904 - 9212010.

Thorsett, S. E., Benjamin, W. R., Brisken, F., Golden, A., and Goss, W. M.: Pulsar PSR B0656+14, the Monogem Ring, and the Origin of the "Knee" in the Primary Cosmic-Ray Spectrum, Astrophys. J., 592, 71 - 73, 2003.

Young, T. R.: A Parameter Study of Type II Supernova Light Curves Using 6 M He Cores, Astrophys. J., 617, 1233 - 1250, 2004. 